\title{
Prevalência de relação sexual e fatores associados em adolescentes escolares de Goiânia, Goiás, Brasil
}

\author{
Prevalence of sexual intercourse and associated factors \\ among adolescents attending schools in Goiânia \\ in the state of Goiás, Brazil
}

\author{
Reinaldo Satoru Azevedo Sasaki ${ }^{1}$ \\ Cláudio Rodrigues Leles ${ }^{2}$ \\ Deborah Carvalho Malta ${ }^{3}$ \\ Luciana Monteiro Vasconcelos Sardinha ${ }^{3}$ \\ Maria do Carmo Matias Freire ${ }^{2}$
}

${ }^{1}$ Departamento de Ginecologia e Obstetrícia, Faculdade de Medicina, Universidade Federal de Goiás (UFG). Pç. Universitária sn/Campus I, Setor Universitário. 74605-220 Goiânia GO Brasil.reinaldo_sasaki@ yahoo.com.br

${ }^{2}$ Faculdade de Odontologia, UFG.

${ }^{3}$ Secretaria de Vigilância em Saúde, Ministério da Saúde.

\begin{abstract}
This study sought to investigate the prevalence of sexual intercourse among adolescents in a Brazilian city and its association with sociodemographic factors, health-risk behaviors, and body image perception. A cross-sectional stu$d y$ was conducted using data from the National Adolescent School-based Health Survey (PeNSE), carried out in 2009. The sample comprised schoolchildren enrolled in the 9th year in the city of Goiânia, Goiás $(n=3,099)$, mostly aged between 13 and 15. The dependent variable was the reporting of sexual intercourse at least once. To identify variables associated with sexual activity, Poisson regression analysis was used. The prevalence of sexual intercourse at least once in the adolescents' lifetime was $26.5 \%$ (95\% CI= 23.8-29.4). Prevalence was higher among males, those aged 14 years or older, who reported not living with their mother or with their father, who attended public schools, reported alcohol, tobacco or other drug use, and were involved in fights and family violen$c e$. The conclusion reached was that the prevalence of sexual intercourse was high and associated with poor sociodemographic conditions, health -risk behaviors and violence. These factors should be considered in the health promotion strategies seeking more safe and healthy sexual behavior during adolescence.
\end{abstract}

Key words Sexual and reproductive health, Adolescent, School health
Resumo Objetivou-se investigar a prevalência de relação sexual em adolescentes de um municipio brasileiro e sua associação com fatores sociodemográficos, comportamentos de risco à saúde e percepção da imagem corporal. Estudo transversal utilizando dados da Pesquisa Nacional de Saúde do Escolar-PeNSE realizada em 2009. A amostra foi composta por escolares do $9^{\circ}$ ano de Goiânia, Goiás ( $n=3.099)$, com predomínio das idades de 13 a 15 anos. A variável dependente foi o relato de relação sexual alguma vez. Para identificação das variáveis associadas foi realizada análise de regressão de Poisson. A prevalência de relação sexual alguma vez foi de $26,5 \%$ (IC95\% = 23,8-29,4). Este comportamento foi mais prevalente em adolescentes do sexo masculino, com idade de 14 anos ou mais, que não moravam com a mãe ou com o pai, estudavam em escolas públicas, relataram ter experimentado álcool, tabaco e outras drogas, $e$ envolvimento em brigas e agressão familiar. Conclui-se que a prevalência de relação sexual alguma vez foi elevada e associada a condições sociodemográficas mais precárias, comportamentos de risco à saúde e violência. Estes fatores devem ser considerados nas estratégias de promoção de saúde, visando comportamentos sexuais seguros e saudáveis na adolescência.

Palavras-chave Saúde sexual e reprodutiva, Adolescente, Saúde do escolar 


\section{Introdução}

A adolescência é uma etapa da vida na qual os futuros padrões de saúde na vida adulta são estabelecidos. Dentre as alterações físicas, psíquicas, comportamentais e sociais que ocorrem na adolescência, o amadurecimento das características sexuais e o início da atividade sexual são transformações importantes. Há evidências de que a idade de início da puberdade tem diminuído e que a idade na qual a maturidade em relação aos papéis sociais é atingida tem aumentado ${ }^{1}$. Assim, o início da vida sexual na adolescência, quando os indivíduos ainda estão em fase de desenvolvimento emocional e cognitivo, pode inseri-los em contextos de vulnerabilidade 2 à gravidez não planejada e às doenças sexualmente transmissíveis/ AIDS $^{3}$.

De acordo com um relatório da Organização Mundial da Saúde, 26\% dos adolescentes na Europa e na América do Norte já haviam iniciado atividade sexual aos 15 anos de idade, e a iniciação sexual precoce estava associada com o não uso ou o uso inadequado de preservativos e suas consequências como gravidez precoce, contágio por doenças sexualmente transmissíveis e pelo vírus da imunodeficiência humana $(\mathrm{HIV})^{4}$. A preocupação com a saúde sexual e reprodutiva de adolescentes e jovens no Brasil se evidenciou num documento do Ministério da Saúde, pautado na concepção de que a garantia dos direitos sexuais e dos direitos reprodutivos desta população é uma questão de direitos humanos e propicia o pleno exercício do direito fundamental à saúde 5 .

Estudos sobre fatores associados ao início da atividade sexual podem ser de grande utilidade para subsidiar políticas de saúde pública com foco mais específico nos problemas evidenciados. De um modo geral, por se sentirem jovens e saudáveis, os adolescentes pouco frequentam os serviços de saúde ${ }^{6}$. Porém, nesta fase, a maioria está frequentando a escola e ainda vivendo no âmbito familiar; portanto, estes são importantes locais onde pode haver orientação para a saúde sexual e reprodutiva. Dentro desta perspectiva, a escola é um espaço privilegiado para a atuação das equipes de saúde ${ }^{7}$.

$\mathrm{Na}$ adolescência, concomitantes com o início da atividade sexual, outros comportamentos trazem risco para a saúde dos indivíduos, como delinquência, afinidade para testar limites e correr riscos, e uso de tabaco, álcool e outras drogas 8 . Embora tenha havido crescente interesse pela questão da sexualidade na adolescência, poucos estudos têm investigado as possíveis associações entre o comportamento sexual e outros comportamentos em saúde que ocorrem nesta fase da vida $^{9-11}$ e há carência de estudos no Brasil ${ }^{12-14}$.

Aspectos da saúde sexual e reprodutiva dos adolescentes foram contemplados no questionário da primeira Pesquisa Nacional de Saúde do Escolar (PeNSE), realizada no Brasil pelo Ministério da Saúde e pelo Instituto Brasileiro de Geografia e Estatística (IBGE) em $2009^{15}$. O estudo mostrou que 30,5\% dos adolescentes brasileiros já tiveram relação sexual alguma vez, sendo mais frequentes entre meninos, de raça/cor preta, com idade acima de 15 anos, estudantes de escolas públicas e cujas mães tinham escolaridade mais bai$\mathrm{xa}^{16}$. Além disso, a prevalência de relação sexual com ou sem uso de preservativo foi associada a aspectos da estrutura familiar, comportamentos de risco à saúde e ausência de orientação sobre gravidez na escola ${ }^{14}$.

Considerando a influência de fatores individuais e das condições de vida na saúde, bem como a coexistência de comportamentos que podem representar riscos à saúde na adolescência, o presente estudo propõe uma análise exploratória buscando identificar outros fatores que podem estar relacionados à atividade sexual de adolescentes escolares. A hipótese é que, além das características individuais e do contexto escolar já pesquisadas ${ }^{14-16}$, a escolaridade da mãe, o envolvimento em brigas e agressão familiar, bem como a percepção da imagem corporal, podem favorecer o início das relações sexuais. Utilizando os dados de um município brasileiro obtidos na PeNSE 2009, e com base em um modelo hierárquico de análise, a presente pesquisa pode contribuir para ampliar o estudo dos determinantes sociais do comportamento sexual em adolescentes. Os resultados podem ser úteis para evidenciar o perfil do adolescente em situação de risco e para subsidiar a elaboração de projetos voltados para a melhoria da saúde dos jovens.

O objetivo deste estudo foi investigar a prevalência de relação sexual em adolescentes escolares de um município brasileiro utilizando os dados da PeNSE 2009 e verificar a sua associação com fatores sociodemográficos, comportamentos de risco à saúde e autoimagem corporal.

\section{Métodos}

\section{Tipo de estudo}

Estudo transversal, utilizando o banco de dados da primeira Pesquisa Nacional de Saúde 
do Escolar - PeNSE, no ano de 2009, coordenada pelo Ministério da Saúde e executada pelo Instituto Brasileiro de Geografia e Estatística $(\mathrm{IBGE})^{15}$. Foram analisados os dados relativos aos adolescentes do município de Goiânia, capital do Estado de Goiás.

A metodologia e os resultados principais da PeNSE encontram-se publicados ${ }^{15,16}$. Foi realizada amostra probabilística de escolares dos períodos diurnos do $9^{\circ}$ ano do ensino fundamental de escolas públicas e privadas das 26 capitais dos estados brasileiros e do Distrito Federal, no ano de 2009, de modo a estimar prevalências de 50\%, com um erro absoluto da ordem de três pontos percentuais e nível de significância de cinco pontos percentuais. Os estudantes presentes no dia da coleta de dados foram convidados a participar da pesquisa e responderam a um questionário estruturado autoaplicável.

\section{Amostra}

No município de Goiânia, participaram da PeNSE 200973 escolas e 112 turmas de escolares, as quais tinham 3.727 estudantes matriculados no $9^{\circ}$ ano naquele ano, com idade entre 11 e 17 anos ou mais, sendo a maioria entre 13 e 15 anos. No dia da pesquisa, 3.291 estudantes estavam presentes e responderam ao questionário e 3.099 responderam à questão: "Você já teve relação sexual (transou) alguma vez?".

\section{Variáveis incluídas}

A variável dependente foi a atividade sexual do adolescente, a partir da questão: "Você já teve relação sexual (transou) alguma vez?", com as categorias de respostas "Sim e Não". As variáveis independentes foram os dados demográficos (sexo, idade, raça/cor autorrelatada), socioeconômicos (escolaridade materna, presença dos pais na residência, dependência administrativa da escola: pública ou privada), comportamentos de risco à saúde (uso de álcool, cigarro e outras drogas alguma vez), violência (envolvimento em brigas e agressão familiar nos últimos 30 dias) e percepção da imagem corporal.

\section{Análise dos dados}

Inicialmente foram calculadas as frequências (porcentagem e intervalo de confiança de 95\%) das variáveis e investigadas associações entre relação sexual e as demais variáveis, por meio de análises bivariadas.
A análise dos fatores associados à prevalência de relação sexual foi realizada levando-se em consideração um modelo hierárquico para determinação do agravo (Figura 1), em quatro blocos. No primeiro bloco situam-se as características demográficas, como sexo, idade e cor/raça; no segundo, condições socioeconômicas, como escolaridade da mãe, morar com a mãe e o pai, e tipo de escola (pública e privada); no terceiro, os comportamentos de risco à saúde, como uso de cigarro, álcool e outras drogas; e, por último, outros fatores associados: violência e percepção da imagem corporal.

Para identificação das variáveis associadas à relação sexual ( sim ou não) foi utilizada a razão de prevalência como medida de efeito com intervalo de confiança de $95 \%$, obtida pela análise de regressão de Poisson. Todas as variáveis com significância menor que 0,20 na análise bivariada foram incluídas no modelo multivariado. Partiuse de um modelo que incluiu todas as variáveis do mesmo bloco, sendo retiradas, uma a uma, as variáveis com valor de $p$ maior que 0,05 . Obtiveram-se três modelos de regressão, nos quais as razões de prevalência foram ajustadas pelas variáveis do mesmo nível ou superiores. Em todas as análises foi considerado o efeito do desenho amostral para amostras complexas, utilizando-se

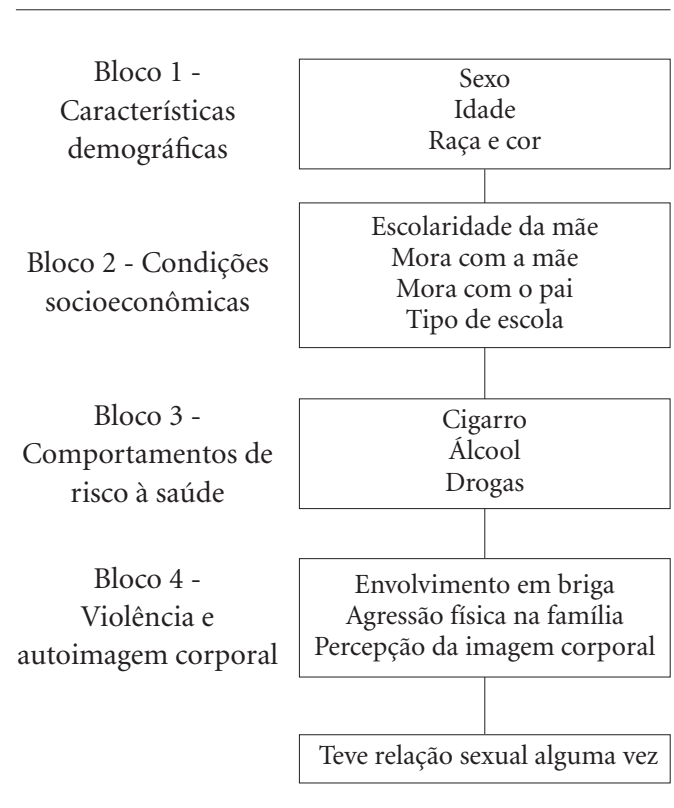

Figura 1. Modelo hierárquico proposto para determinação da relação sexual em adolescentes escolares do município de Goiânia. Pesquisa Nacional de Saúde do Escolar (PeNSE), Brasil, 2009. 
pesos amostrais calculados no módulo Complex Samples do programa estatístico SPSS 17.0. Em todas as análises descritivas e inferenciais foi utilizado o programa Stata 9.1 (Stata Corp., College Station, TX, USA).

\section{Aspectos éticos}

O projeto da PeNSE foi aprovado pela Comissão Nacional de Ética em Pesquisa - CONEP.

\section{Resultados}

A Tabela 1 descreve as características demográficas e socioeconômicas dos participantes, sendo predominantes os adolescentes do sexo feminino $(51,6 \%)$, na faixa etária de 14 anos $(49,8 \%)$ e

Tabela 1. Características demográficas e socioeconômicas dos adolescentes do município de Goiânia. Pesquisa Nacional de Saúde Escolar (PeNSE), Brasil, 2009.

\begin{tabular}{|c|c|}
\hline Variáveis & $\begin{array}{c}\text { Frequência } \\
\text { n (\% válidos) }\end{array}$ \\
\hline \multicolumn{2}{|l|}{ Demográficas } \\
\hline \multicolumn{2}{|l|}{ Sexo $(n=3099)$} \\
\hline Feminino & $1.599(51,6)$ \\
\hline Masculino & $1.500(48,4)$ \\
\hline \multicolumn{2}{|l|}{ Idade $(\mathrm{n}=3095)$} \\
\hline$\leq 13$ anos & $921(29,7)$ \\
\hline 14 anos & $1.543(49,8)$ \\
\hline$\geq 15$ anos & $631(20,4)$ \\
\hline \multicolumn{2}{|l|}{ Raça/cor $(n=3049)$} \\
\hline Branca & $1.189(39,0)$ \\
\hline Preta & $326(10,7)$ \\
\hline Parda & $1.253(41,1)$ \\
\hline Oriental (amarela) & $162(5,3)$ \\
\hline Indígena & $119(3,9)$ \\
\hline \multicolumn{2}{|l|}{ Socioeconômicas } \\
\hline \multicolumn{2}{|c|}{ Tipo de escola $(\mathrm{n}=3099)$} \\
\hline Privada & $981(31,7)$ \\
\hline Pública & $2.118(68,3)$ \\
\hline \multicolumn{2}{|c|}{ Escolaridade da mãe $(\mathrm{n}=2525)$} \\
\hline$<9$ anos & $752(29,8)$ \\
\hline $9-11$ anos & $416(16,5)$ \\
\hline$\geq 12$ anos & $1.357(53,7)$ \\
\hline \multicolumn{2}{|c|}{ Mora com a mãe $(\mathrm{n}=3067)$} \\
\hline Sim & $2.684(87,5)$ \\
\hline Não & $383(12,5)$ \\
\hline \multicolumn{2}{|c|}{ Mora com o pai $(\mathrm{n}=3072)$} \\
\hline Sim & $1.846(60,1)$ \\
\hline Não & $1.226(39,9)$ \\
\hline
\end{tabular}

de cor parda $(41,1 \%)$. A maioria dos estudantes $(68,3 \%)$ era de escola pública, morava com a mãe $(87,5 \%)$ e com o pai $(60,1 \%)$. Mais da metade das mães dos adolescentes (53,7\%) tinham 12 anos ou mais de estudo.

A prevalência de relação sexual alguma vez foi de 26,5\% (IC 95\%=23,8-29,4). Nas Tabelas 2 e 3 encontram-se as medidas da razão de prevalência não ajustadas das variáveis relacionadas com a prevalência de relação sexual alguma vez. Os resultados mostram que os meninos apresentaram prevalência 2,52 vezes maior que as meninas ( $\mathrm{p}$ $<0,001$ ). Indivíduos de cor preta apresentaram maior prevalência de relação sexual em comparação com brancos (RP = 1,29; IC95\%: 1,06-1,58). Este comportamento foi também mais frequente na faixa etária de 15 anos ou mais, entre os adolescentes que não moravam com a mãe ou com o pai e entre os estudantes de escolas públicas ( $\mathrm{p}$ $<0,001$ ). A escolaridade materna inicialmente demonstrou efeito protetor significativo, sendo menor a prevalência de relação sexual entre os adolescentes cujas mães tinham escolaridade mais alta (12 anos ou mais).

Em relação aos comportamentos de risco à saúde e violência (Tabela 3), houve associação significativa com o uso de cigarro, álcool e drogas, bem como com envolvimento em brigas e episódios de agressão familiar nos últimos 30 dias $(\mathrm{p}<0,001)$. O relato de relação sexual foi associado também à autoimagem corporal, com efeito protetor para adolescentes que se julgavam gordos ou muito gordos $(\mathrm{p}=0,035)$.

Os resultados da análise de regressão múltipla de Poisson estão na Tabela 4. Após ajuste das variáveis do bloco 1 (Figura 1), as variáveis sexo e idade apresentaram associação com a ocorrência de relação sexual, não havendo associação desta com a raça/cor do adolescente $(\mathrm{p}=0,173)$. No bloco 2, a variável escolaridade materna foi removida, permanecendo associação com não morar com a mãe, não morar com o pai e estudar em escola pública. No bloco 3 todas as variáveis permaneceram associadas. No bloco 4, permaneceram associadas as variáveis envolvimento em brigas e agressão familiar. Dentre todas as variáveis incluídas no modelo final, as maiores prevalências foram verificadas em adolescentes de 15 anos ou mais, que informaram ter experimentado álcool pelo menos alguma vez e ser do sexo masculino. A prevalência de relação sexual foi $76 \%$ maior nos que experimentaram cigarros alguma vez, $63 \%$ maior nos que se envolveram em brigas, $47 \%$ maior naqueles que experimentaram drogas e 38\% maior naqueles que relataram agressão fí- 
Tabela 2. Prevalência de relação sexual alguma vez na vida segundo variáveis demográficas e socioeconômicas, em adolescentes do município de Goiânia. Pesquisa Nacional de Saúde Escolar (PeNSE), Brasil, 2009.

\begin{tabular}{|c|c|c|c|}
\hline Variáveis & $\begin{array}{c}\text { Prevalência de } \\
\text { relação sexual } \\
\%(\mathrm{IC} 95 \%)^{*}\end{array}$ & RP $(\mathrm{IC} 95 \%)^{*}$ & $\mathbf{p}^{* *}$ \\
\hline \multicolumn{4}{|l|}{ Demográficas } \\
\hline \multicolumn{4}{|l|}{ Sexo $(n=3099)$} \\
\hline Feminino & $15,2(13,3-17,1)$ & \multirow{2}{*}{$2,52(2,18-2,90)$} & \multirow[t]{2}{*}{$<0,001$} \\
\hline Masculino & $38,3(35,7-40,9)$ & & \\
\hline \multicolumn{4}{|l|}{ Idade $(\mathrm{n}=3095)$} \\
\hline$\leq 13$ anos & $12,8(10,4-15,2)$ & \multirow{3}{*}{$\begin{array}{l}1,85(1,50-2,29) \\
4,15(3,39-5,09)\end{array}$} & \multirow[t]{3}{*}{$<0,001$} \\
\hline 14 anos & $23,7(21,5-25,9)$ & & \\
\hline$\geq 15$ anos & $53,1(48,9-57,2)$ & & \\
\hline \multicolumn{4}{|l|}{ Raça/cor $(\mathrm{n}=3049)$} \\
\hline Branca & $24,8(22,2-27,5)$ & \multirow{5}{*}{$\begin{array}{c}1 \\
1,29(1,06-1,58) \\
1,06(0,92-1,23) \\
1,01(0,74-1,37) \\
1,08(0,78-1,51)\end{array}$} & \multirow[t]{5}{*}{0,173} \\
\hline Preta & $32,0(26,6-37,5)$ & & \\
\hline Parda & $26,4(23,8-28,9)$ & & \\
\hline Oriental (amarela) & $25,1(17,8-32,4)$ & & \\
\hline Indígena & $26,9(18,4-35,4)$ & & \\
\hline \multicolumn{4}{|l|}{ Socioeconômicas } \\
\hline \multicolumn{4}{|c|}{ Escolaridade da mãe $(\mathrm{n}=2525)$} \\
\hline$<9$ anos & $32,5(29,0-36,1)$ & \multirow{3}{*}{$\begin{array}{l}0,87(0,71-1,05) \\
0,70(0,60-0,82)\end{array}$} & \multirow[t]{3}{*}{$<0,001$} \\
\hline $9-11$ anos & $28,2(23,6-32,8)$ & & \\
\hline$\geq 12$ anos & $22,9(20,4-25,3)$ & & \\
\hline \multicolumn{4}{|c|}{ Mora com a mãe $(\mathrm{n}=3067)$} \\
\hline Sim & $24,8(23,1-26,6)$ & \multirow{2}{*}{$1,46(1,24-1,71)$} & \multirow{2}{*}{$<0,001$} \\
\hline Não & $36,4(31,1-41,6)$ & & \\
\hline \multicolumn{4}{|c|}{ Mora com o pai $(\mathrm{n}=3072)$} \\
\hline Sim & $23,8(21,7-25,9)$ & \multirow{2}{*}{$1,30(1,14-1,47)$} & \multirow[t]{2}{*}{$<0,001$} \\
\hline Não & $30,8(28,1-33,6)$ & & \\
\hline \multicolumn{4}{|c|}{ Tipo de escola $(\mathrm{n}=3099)$} \\
\hline Privada & $18,8(16,3-21,4)$ & \multirow{2}{*}{$1,61(1,38-1,88)$} & \multirow{2}{*}{$<0,001$} \\
\hline Pública & $30,4(28,3-32,6)$ & & \\
\hline
\end{tabular}

IC95\%: intervalo de 95\% de confiança; RP: razão de prevalência bruta. " Corrigido para o desenho amostral. *Teste de Wald.

sica familiar. Não morar com a mãe aumentou a prevalência de relação sexual em $21 \%$ e não morar com o pai, aumentou em $20 \%$.

\section{Discussão}

O presente estudo mostra que o comportamento sexual dos adolescentes está associado a outros comportamentos que se estabelecem nesta etapa da vida. Mostra ainda desigualdades de gênero e socioeconômicas na prática da relação sexual. Estes achados sugerem que há um padrão de comportamento que caracteriza o início da vida sexual na adolescência, resultante da interação entre fatores individuais, condições de vida, questões familiares e exposição a fatores de risco à saúde, os quais merecem atenção. Destaca-se a relação entre relação sexual e condição socioeconômica mais baixa, situações de violência, bem como comportamentos desfavoráveis como uso de ál$\mathrm{cool}$, tabagismo e outras drogas. O delineamento transversal do estudo, entretanto, não permite esclarecer relações de causa-efeito.

Os resultados devem ser interpretados considerando-se os aspectos metodológicos do estudo, bem como suas limitações. Foram utilizados dados de inquérito nacional, sendo o primeiro estudo a avaliar a prevalência de relação sexual e diversos fatores associados em um município brasileiro com base nestes dados. O instrumento é constituído de questões validadas sobre uma ampla gama de fatores de risco e proteção para a saúde do adolescente escolar, sendo que o tamanho da amostra permite inferência para a população escolar da capital do Estado de Goiás.

Pesquisas no ambiente escolar possibilitam que os resultados sejam utilizados na indução de 


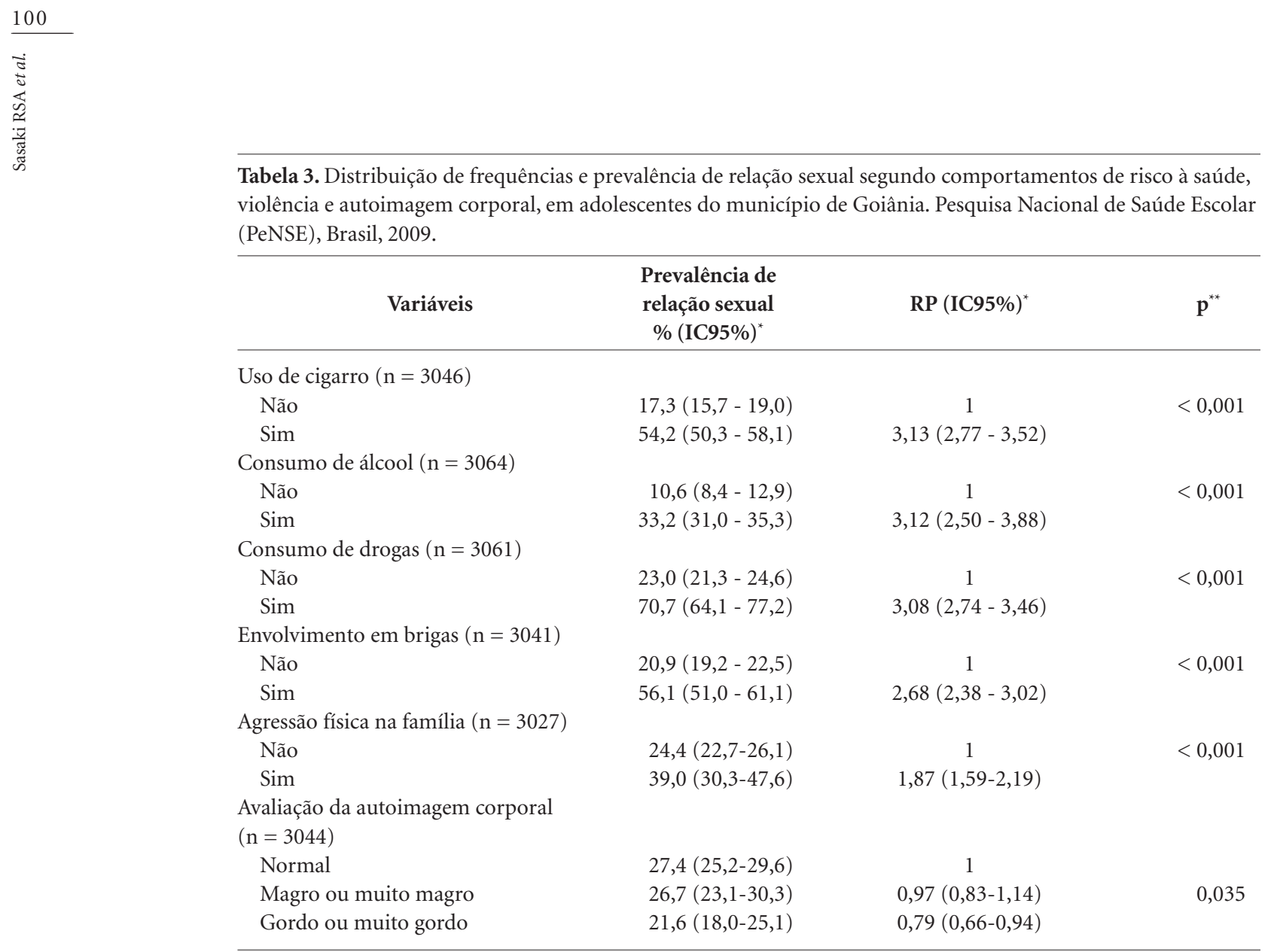

IC95\%: intervalo de 95\% de confiança; RP: razão de prevalência bruta. ${ }^{*}$ Corrigido para o desenho amostral. ${ }^{*}$ Teste de Wald.

políticas públicas de promoção à saúde e prevenção para o público específico. Algumas considerações devem ser feitas quanto à amostra, pois ela se limita aos estudantes com frequência regular na escola, portanto, excluindo os adolescentes fora do sistema educacional regular. Contudo, no Brasil, a cobertura do sistema de ensino, quando se consideram adolescentes mais jovens, tem sido alta. Em 2007, ano que serviu de base para o cálculo amostral da PeNSE ${ }^{17}$, 97\% dos indivíduos entre 10 e 14 anos encontravam-se matriculados em escolas. Também não estão contemplados nesta amostra os estudantes dos cursos noturnos. Estes podem diferir daqueles do turno diurno, pois frequentemente são estudantes de situação econômica inferior, com objetivos diferentes, inseridos no mercado de trabalho, com maior taxa de abandono das escolas e muitas vezes com educação de pior qualidade ${ }^{18}$. Outro aspecto a ser considerado é que a amostra da PeNSE é de alunos matriculados no $9^{\circ}$ ano, predominando alunos entre 13 a 15 anos. Esta diferença de idade resulta em diferentes oportunidades para iniciar a atividade sexual. Alunos mais velhos têm mais chances de se expor à relação sexual e esta pode estar associada a outros fatores de interesse na presente análise. Consideramos ainda que no questionário não havia perguntas sobre a gestação e partos, limitando a análise dos aspectos reprodutivos.

A prevalência de relação sexual em Goiânia $(26, \%)$ foi inferior à verificada na amostra nacional da PeNSE 2009 (30,5\%), mas encontra-se numa posição intermediária quando comparada com as demais capitais ${ }^{16}$. Os resultados das análises bivariadas da relação entre a relação sexual e as variáveis sociodemográficas estão de acordo com a amostra nacional ${ }^{14,16}$. Contudo, após a análise de regressão múltipla, quando os diversos fatores foram analisados em conjunto, a associação com a raça/cor do estudante e escolaridade da mãe não permaneceram significantes, em concordância com estudo realizado em outra localidade brasileira ${ }^{19}$. Estas relações se manifestam de forma diferente quando se compara dados de outros países. Nos Estados Unidos, o Sistema de Vigilância de Risco e Comportamento da Juventude no ano de 2009 não encontrou diferença de gênero na presença de intercurso sexual $^{20}$, enquanto outros estudos mostraram 
Tabela 4. Resultado da análise de regressão de Poisson da associação entre relação sexual e variáveis independentes, em adolescentes do município de Goiânia. Pesquisa Nacional de Saúde Escolar (PeNSE), Brasil, 2009.

\begin{tabular}{|c|c|c|c|c|c|}
\hline Blocos & Variáveis & $\begin{array}{c}\text { Modelo I } \\
\text { RP (IC95\%) }\end{array}$ & $\begin{array}{c}\text { Modelo II } \\
\text { RP (IC95\%) }\end{array}$ & $\begin{array}{c}\text { Modelo III } \\
\text { RP (IC95\%) }\end{array}$ & $\begin{array}{c}\text { Modelo IV } \\
\text { RP (IC95\%) }\end{array}$ \\
\hline \multirow[t]{7}{*}{1} & Sexo & & & & \\
\hline & Feminino & 1 & & & \\
\hline & Masculino & $2,22(1,93-2,55)$ & & & \\
\hline & Idade & & & & \\
\hline & $\leq 13$ anos & 1 & & & \\
\hline & 14 anos & $1,80(1,46-2,21)$ & & & \\
\hline & $\geq 15$ anos & $3,61(2,95-4,43)$ & & & \\
\hline \multirow[t]{9}{*}{2} & Tipo de escola & & & & \\
\hline & Privada & & 1 & & \\
\hline & Pública & & $1,35(1,14-1,59)$ & & \\
\hline & Mora com a mãe & & & & \\
\hline & Sim & & 1 & & \\
\hline & Não & & $1,21(1,03-1,44)$ & & \\
\hline & Mora com o pai & & & & \\
\hline & Sim & & 1 & & \\
\hline & Não & & $1,20(1,05-1,38)$ & & \\
\hline \multirow[t]{9}{*}{3} & Consumo de álcool & & & & \\
\hline & Não & & & 1 & \\
\hline & Sim & & & $2,30(1,78-2,97)$ & \\
\hline & Uso de cigarro & & & & \\
\hline & Não & & & 1 & \\
\hline & Sim & & & $1,76(1,52-2,03)$ & \\
\hline & Consumo de drogas & & & & \\
\hline & Não & & & 1 & \\
\hline & Sim & & & $1,47(1,25-1,72)$ & \\
\hline \multirow[t]{6}{*}{4} & Envolvimento em brigas & & & & \\
\hline & Não & & & & 1 \\
\hline & Sim & & & & $1,63(1,40-1,89)$ \\
\hline & Agressão física na família & & & & \\
\hline & Não & & & & 1 \\
\hline & Sim & & & & $1,38(1,14-1,67)$ \\
\hline
\end{tabular}

IC95\%: intervalo de 95\% de confiança; RP: razão de prevalência

que a raça negra, em combinação com a renda e a estrutura familiar, interferem na prevalência deste comportamento ${ }^{21,22}$. O efeito da condição socioeconômica confirma os achados de outros estudos $^{12,19,21,22}$, mas foi diferente daquele verificado na Colômbia, onde a relação sexual foi mais prevalente em adolescentes de famílias de renda $\operatorname{alta}^{11}$.

Os resultados em relação à idade, sexo e estrutura familiar corroboram os estudos anteriores e mostram que a idade mais avançada e ser do sexo masculino ${ }^{12,14.19,23}$, bem como a estrutura familiar ${ }^{12,14,19,21,22,24}$, são fatores que aumentam as chances de ter relação sexual. A família atua como fator de proteção para relação sexual precoce e também para atitudes de risco, como o uso de tabaco, álcool e drogas ${ }^{25}$. Segundo a pesquisa
Voz dos Adolescentes, realizada no Brasil entre os anos de 2003 e 2007, mais de 90\% dos adolescentes referiram ter na família sua principal referência, e o recebimento de apoio e limites era visto como uma forma de cuidado para com eles ${ }^{26}$. Um estudo realizado em adultos na Eslovênia mostrou que as mulheres que adquiriram a maior parte dos conhecimentos dos pais ou na escola, iniciaram a atividade sexual mais tarde, quando estavam mais preparados para práticas sexuais mais seguras ${ }^{27}$.

A associação encontrada entre a prevalência de relação sexual alguma vez e o uso de tabaco, álcool ou outras drogas alguma vez está em concordância com diversos estudos anteriores ${ }^{11-13,23,28}$ e foi verificada também na amostra nacional da PeNSE $2009^{14}$. A utilização de substâncias psico- 
ativas, principalmente de álcool e drogas ilícitas, expõe os adolescentes a situações de violência, risco de gestação indesejada e de aquisição de doenças sexualmente transmissíveis, segundo relatório da UNICEF ${ }^{29}$. Revisões de literatura sobre o tema corroboram esta afirmação, mostrando que o consumo de álcool está relacionado à primeira relação sexual e a comportamentos sexuais de risco, tais como múltiplos parceiros e relações casuais $^{9,10}$, e prática de sexo sem preservativo ${ }^{30}$. Contudo, não há consenso em relação à influência causal do álcool no uso de preservativos masculinos. Um estudo de abordagem qualitativa em adolescentes de escola pública no Brasil mostrou que estes reconhecem o risco entre o consumo de álcool e o comportamento sexual, destacam que o ato de beber facilita as relações entre os pares e apontam a influência da mídia neste processo ${ }^{31}$.

A influência de situações relacionadas à violência na prevalência de relação sexual tem sido demonstrada em outros estudos, os quais mostram que a violência tanto intra quanto extrafamiliar presentes em sociedades complexas está associada aos comportamentos de risco sexuais e não sexuais, ampliando a vulnerabilidade dos adolescentes ${ }^{12,32}$. Neste estudo, não foi encontrada associação entre o relato de relação sexual e a percepção da imagem corporal, estando de acordo com estudo anterior sobre insatisfação corporal e início da atividade sexual ${ }^{33}$.

A presente investigação fornece um perfil do adolescente escolar em relação ao seu comportamento sexual e condições socioeconômicas, hábitos de vida e situações de violência. Os resultados podem ser úteis para que os pais, educadores e profissionais da saúde possam identificar aqueles com maior chance de iniciar ou ter iniciado vida sexual e promover ações educativas para evitar que, nesta fase de profundas mudanças, os prováveis riscos decorrentes da relação sexual sejam minimizados.

A escola constitui um espaço adequado para o desenvolvimento de programas de promoção de saúde voltados para crianças e adolescentes ${ }^{34}$. A maior parte dos escolares da PeNSE 2009 revelaram ter recebido orientações nas escolas quanto à saúde sexual, DST/AIDS, prevenção de gravidez e aquisição gratuita de preservativos, as quais podem contribuir para a mudança de comportamentos relacionados à sexualidade ${ }^{35,36}$.

Conclui-se que a prevalência de relação sexual alguma vez nos adolescentes escolares foi elevada e associada a fatores sociodemográficos, comportamentos de risco à saúde e situações de violência entre os adolescentes pesquisados. Estes fatores devem, portanto, ser considerados em conjunto no planejamento e na avaliação de estratégias de promoção de saúde voltadas para este grupo populacional, buscando a implementação de medidas mais apropriadas e efetivas. Ações integradas de educação sexual e prevenção do tabagismo, álcool e outras drogas, bem como da violência familiar e extrafamiliar têm, portanto, $\mathrm{o}$ potencial de contribuir para um comportamento sexual mais seguro e saudável na adolescência. Há, ainda, a necessidade de mais estudos buscando elucidar os mecanismos que explicam as associações observadas no presente estudo, analisando-se a influência dos aspectos psicossociais do comportamento sexual, tais como a natureza das relações amorosas e suas dimensões. 


\section{Colaboradores}

RSA Sasaki participou da concepção do estudo, analisou os dados e redigiu o artigo. CR Leles participou da concepção do estudo, colaborou na metodologia, análise estatística dos dados e revisou o artigo. DC Malta e LMV Sardinha participaram da concepção do estudo, atuaram no projeto que originou o banco de dados (PeNSE 2009) e revisaram o artigo. MCM Freire foi orientadora do estudo e revisou o artigo.

\section{Referências}

1. Sawyer M, Afifi RA, Bearinger LH, Blakemore SJ, Dick B, Ezeh AC, Patton GC. Adolescence: a foundation for future health. Lancet 2012; 379:1630-1640.

2. Sánchez AIM, Bertolozzi MR. Pode o conceito de vulnerabilidade apoiar a construção do conhecimento em Saúde Coletiva? Cien Saude Colet 2007; 12(2):319-324.

3. Cruzeiro ALS, Souza LDM, Silva RA, Pinheiro RT, Rocha CLA, Horta BL. Comportamento sexual de risco: fatores associados ao número de parceiros sexuais e ao uso de preservativo em adolescentes. Cien Saude Colet 2010; 15(1):1149-1158.

4. Currie C, Zanotti C, Morgan A, Currie D, Looze M, Roberts C, Samdal O, Smith ORF, Barnekow V. Social determinants of health and well-being among young people: Health Behaviour in School-Aged Children (HBSC) study: international report from the 2009/2010 survey. Copenhagen: WHO Regional Office for Europe; 2012. (Health Policy for Children and Adolescents, No. 6).

5. Brasil. Ministério da Saúde (MS). Secretaria de Atenção à Saúde. Departamento de Ações Programáticas Estratégicas. Marco teórico e referencial: saúde sexual e saúde reprodutiva de adolescentes e jovens. Brasília: MS; 2006.

6. Castro MG, Abramovay M, Silva LB. Juventude e sexualidade. Brasília: UNESCO Brasil; 2004.

7. Demarzo MMP, Aquilante AG. Saúde escolar e escolas promotoras de saúde. In: Programa de Atualização em Medicina de Família e Comunidade. Porto Alegre: Artmed/Pan-Americana; 2008. Vol. 3.

8. Heaven PCL. Adolescent health - The role of individual differences. London: Routledge; 1996.

9. Halpern-Felsher BL, Millstein SG, Ellen JM. Relationship of alcohol use and risky sexual behavior: a review and analysis of findings. J Adolesc Health 1996; 19(5):331-336.

10. Cooper ML. Alcohol use and risky sexual behavior among college students and youth: evaluating the evidence. J Stud Alcohol 2002; 14(Supl.):101-117.

11. Campo-Arias A, Ceballo A, Herazo E. Prevalência do padrão de comportamento de risco para a saúde sexual e reprodutiva em estudantes adolescentes. Rev Latino -Am Enfermagem 2010; 18(2):170-174.

12. Cruzeiro ALS, Souza LDDEM, Silva RADA, Horta BL, Muenzer RM, Faria AD. Iniciação sexual entre adolescentes de Pelotas, Rio Grande do Sul. Rev Bras Crescimento Desenvolv Hum 2008; 18(2):116-125.

13. Miozzo L, Dalberto ER, Silveira DX, Terra MB. Consumo de substâncias psicoativas em uma amostra de adolescentes e sua relação com o comportamento sexual. J Bras Psiquiatr 2013; 62(2):93-100.

14. Oliveira-Campos M, Giatti L, Malta D, Barreto SM. Contextual factors associated with sexual behavior among Brazilian adolescents. Ann Epidemiol 2013; 23(10):629-635.

15. Instituto Brasileiro de Geografia e Estatística (IBGE). Pesquisa Nacional de Saúde do Escolar. Rio de Janeiro: IBGE; 2009.

16. Malta DC, Silva MAI, Melo FCM, Monteiro RA, Porto DL, Sardinha, LMV, Freitas PC. Saúde sexual dos adolescentes segundo a Pesquisa Nacional de Saúde dos Escolares. Rev Bras Epidemiol 2011; 14(1):147-156. 
17. Instituto Brasileiro de Geografia e Estatística (IBGE). Pesquisa Nacional por amostra de domicílio. PNAD 2009. [página na internet]. [acessado $2012 \mathrm{dez} 20$ ]. Disponível em: http://www.ibge.gov.br/home/estatistica / populacao/trabalhoerendimento/pnad2009/default. shtm. html

18. Togni AC, Carvalho MJS. A escola noturna de ensino médio no Brasil. Rev Iberoamer Educación 2007; 44:6176.

19. Borges ALV, Latorre MRDO, Schor N. Fatores associados ao início da vida sexual de adolescentes matriculados em uma unidade de saúde da família da zona leste do Município de São Paulo, Brasil. Cad Saude Publica 2007; 23(7):1583-1594.

20. Youth Risk Behavior Survey (YRBS). Trends in the prevalence of sexual behavior. National YRBS: 1991-2009. Atlanta, GA: Centers for Disease Control and Prevention; 2009. [página na internet]. [acessado $2012 \mathrm{fev}$ 01]. Disponível em: http://www.cdc.gov/healthyyouth/ yrbs/pdf/us_sexual_trend_yrbs.pdf>

21. Blum RW, Beuhring T, Shew ML, Bearinger LH, Sieving $\mathrm{RE}$, Resnick MD. The effects of race/ethnicity, income, and family structure on adolescent risk behaviors. Am J Public Health 2000; 90(12):1879-1884.

22. Santelli JS, Lowry R, Brener ND, Robin L. The association of sexual behaviors with socioeconomic status, family structure and race/ethnicity among US adolescents. Am J Public Health 2000; 90(10):1582-1588.

23. Rudatsikira E, Ogwell AE, Siziya S, Muula AS. Prevalence of sexual intercourse among school-going adolescents in Coast Province, Kenya. Tanzan Health Res Bull 2007; 9(3):159-163.

24. Peres CA, Rutherford G, Borges G, Galano E, Hudes ES, Hearst N. Family structure and adolescent sexual behavior in a poor area of Sao Paulo, Brazil. J Adolesc Health 2008; 42(2):177-183.

25. Malta DC, Porto DL, Melo FCM, Monteiro RA, Sardinha LMV, Lessa BH. Família e proteção ao uso de tabaco, álcool e drogas em adolescentes, Pesquisa Nacional de Saúde dos Escolares. Rev Bras Epidemiol 2011; 14(Supl. 1):166-177.

26. Fundo das Nações Unidas para a Infância (UNICEF), Instituto Ayrton Senna (IAS), Fundação Itaú Social (FIAS). Adolescentes e jovens do Brasil: participação social e politica. Brasília: UNICEF; 2008.

27. Klavs I, Rodrigues LC, Weiss HA, Hayes R. Factors associated with early sexual debut in Slovenia: results of a general population survey. Sex Transm Infect 2006; 82(6):478-483.

28. Strauch ES, Pinheiro RT, Silva RA, Horta BL. Alcohol use among adolescents: a population-based study. Rev Saude Publica 2009; 43(4):647-655.
29. Fundo das Nações Unidas para a Infância (Unicef). $O$ direito de ser adolescente: Oportunidade para reduzir vulnerabilidades e superar desigualdades. Brasília: UNICEF; 2011.

30. Cardoso LRD, Malbergier A, Figueiredo TFB. O consumo de álcool como fator de risco para a transmissão das DSTs/HIV/Aids. Rev Psiq Clín 2008; 35(Supl. 1):70-75.

31. Sampaio Filho FJL, Sousa PRM, Vieira NFC, Nóbrega MFB, Gubert FA, Pinheiro PNC. Percepção de risco de adolescentes escolares na relação consumo de álcool e comportamento sexual. Rev Gaúcha Enferm 2010; 31(3):508-514.

32. Teixeira SAM, Taquette SR. Violência e atividade sexual desprotegida em adolescentes menores de 15 anos. Rev Assoc Med Bras 2010; 56(4):440-446.

33. Langoni PO, Aerts DRGC, Alves GG, Câmara SG. Prevalência de insatisfação com a imagem corporal e fatores associados em adolescentes escolares. Universidade Luterana do Brasil - RS - Grupo Hospitalar Conceição/ GHC - Brasil, 2010. [página na Internet]. [acessado 2011 abr 19]. Disponível em: http://www.fef.unicamp .br/hotsites/imagemcorporal2010/cd/anais/trabalhos/ portugues/Area3/IC3-37.pdf.

34. Andrade HHSM, Mello MB, Sousa MH, Makuch MY, Bertoni N, Faúndes A. Changes in sexual behavior following a sex education program in Brazilian public schools. Cad Saude Publica 2009; 25(5):1168-1176.

35. Malta DC, Sardinha LMV, Brito I, Gomes MRO, Rabelo M, Morais Neto OL, Penna GO. Orientações de saúde reprodutiva recebidas na escola - uma análise da Pesquisa Nacional de Saúde do Escolar nas capitais brasileiras e no Distrito Federal, 2009. Epidemiol Serv Saúde 2011; 20(4):481-490.

36. Berquó E, Garcia S, Lima L. Reprodução na juventude: perfis sociodemográficos, comportamentais e reprodutivos na PNDS 2006. Rev Saude Publica 2012; 46(4):685-693.

Artigo apresentado em 26/05/2014

Aprovado em 20/06/2014

Versão final apresentada em 22/06/2014 